\title{
CONFECCIÓN DE UN LÉXICO JURÍDICO GRIEGO
}

\begin{abstract}
A Greek juridical lexicon is being made at Granada University starting from the lexical materials provided basically by Attic orators and Inscriptions. The first contribution to this project has been the work Juridical Lexicon of Demosthenes (Granada 1988). This juridical lexicon is understood on a very wide sense, so, it includes different levels of the Greek social life (economics, family, politics, religion), and it tries to be a useful instrument for the linguist as well as for the jurist.
\end{abstract}

\section{Consideraciones generales}

Una de las lagunas más llamativas de la Filología Clásica es la ausencia de un léxico jurídico de la lengua griega. $\mathrm{Ni}$ en su vertiente de lenguaje técnico ni en la de diccionario de autor registramos trabajo alguno, salvo las esporádicas referencias en los manuales enciclopédicos o los estudios concretos sobre ciertos términos. Un desinterés que quizá haya que encuadrar dentro del general abandono que los oradores, fuente principal para un estudio de este tipo, han sufrido en nuestro siglo. Pues bien, con nuestra tesis doctoral, Léxico juridico de Demóstenes (Granada 1988), tratamos de cubrir parcialmente este vacío, y precisamente con un autor que, aunque es conocido sólo fragmentariamente en esta faceta a través de los tratados clásicos de Derecho griego, ocupa un lugar importante, tanto por el número de sus discursos como por el alto porcentaje de léxico jurídico total que contienen, que podemos calcular en un 80 por 100 .

De otra parte, este trabajo se enmarca dentro de un proyecto general más amplio, que pretende confeccionar un léxico jurídico completo del griego ático con base fundamentalmente en los oradores y las inscripciones, y crear de esta forma un instrumento capaz de interesar tanto al filólogo como al jurista, posibilitando el acercamiento de las disciplinas que ambos representan. Se está realizando en la Universidad de Granada por un equipo del que formamos parte, digirido por el Dr. Calvo Martínez. 
Hemos adoptado para nuestro trabajo la forma de léxico por parecernos las más completa, pues, además de incluir la traducción, permite simplificar los contextos y distribuir los artículos de acuerdo con criterios sintácticos y semánticos. Pero los diccionarios o léxicos de autor no son una creación reciente de la lexicografia. Ya los mismos griegos sintieron la necesidad, desde época temprana ', de crear léxicos o glosarios, al no comprender ciertas palabras. Con todo, es en el siglo xIX y, sobre todo, en el xx cuando se comienzan a hacer léxicos, indices y concordancias de forma más sistemática y científica. De léxico jurídico ya hemos dicho que no existe ningún diccionario, y de nuestro autor en cuestión, Demóstenes, sólo registramos, además del genérico Índice de los oradores áticos de Reiske, el Index Demosthenicus de S. Preuss (Leipzig 1892), que no incluye los documentos insertados en los discursos (leyes, decretos, etc.), y el abreviado índice de términos, más institucionales que jurídicos, que incorpora R. Weil al final del volumen IV de Plaidoyers civils (Collection Budé, pp. 128-190, París 1960).

\section{Selección de términos}

Una de las mayores dificultades al abordar un léxico jurídico es delimitar su ámbito. La línea divisoria resulta fluctuante, debido en gran parte a la ausencia en el Derecho griego, a diferencia del romano y los actuales, de un corpus específico y concreto de términos, sin negar por ello que los cimientos de su formación estuviesen ya puestos. A nivel común, lo propiamente jurídico serían las actividades relacionadas con el juicio, o sea, el derecho procesal. Pero igualmente podríamos ampliar el concepto a otros ámbitos de la vida social: economía (contratos, préstamos, hipoteca, intereses...), familia (casamiento, divorcio, dote, sucesión, testamento...), religión (el juramento, tan imprescindible en cualquier acto jurídico), o, sobre todo, política, donde las magistraturas desempeñaban a la vez funciones políticas y jurídicas, al no estar bien delimitada la división de poderes. Difícilmente encontraremos actividad que no esté reglada jurídicamente. Pero, ¿dónde acaba un concepto y comienza el otro? Hemos tratado de entender lo jurídico en sentido amplio, con exclusión de lo estrictamente moral o religioso. Incluso hemos

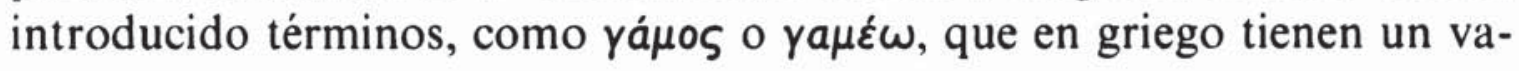

1 Con detalle, v. F. R. Adrados, E. Gangutia, J. López Facal y C. Serrano Aybar, Introducción a la Lexicografia griega, Madrid 1977. 
lor meramente festivo, pero que en nuestra lengua adoptan una connotación jurídica (casamiento, casarse).

Un segundo tipo de selección implica a la palabra misma. Consiste en discernir el uso común del especializado. Y esto con frecuencia no se marca en los grandes diccionarios con un apartado propio. Menos información encontramos en los grandes diccionarios enciclopédicos de la Antigüedad ( $R E$, el Daremberg-Saglio, Artemis, The Oxford Classical Dictionary, etc.) o en las nuevas enciclopedias juridicas (sobre todo, el Digesto Italiano y sus dos posteriores ediciones) sobre el aspecto cronológico del término, en el sentido de si el uso común precedió al técnico o al contrario; sincrónicamente tampoco nos ofrecen más datos, y así se soslaya que en un momento dado un mismo término pueda tener en una polis un valor técnico concreto y en otra un valor puramente común.

El tema de la creación del vocabulario jurídico en Grecia, que escapa a nuestro objeto de estudio, ha sido estudiado por el Dr. Lucas de Dios en su artículo "Consideraciones generales sobre el vocabulario jurídico griego" (Epos 2, 1986, pp. 187-204). Establece tres fases; en la primera encontramos una serie de términos que tienen un significado

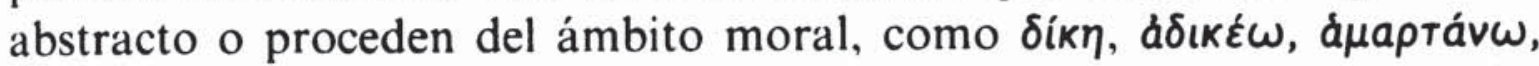

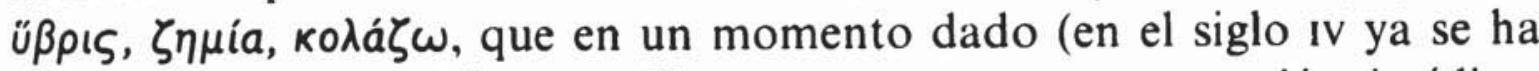
producido este cambio) se utilizan para expresar una noción jurídica. En una segunda fase aparecen los neologismos exclusivamente jurídicos, creados para cubrir las nuevas realidades legales; para su formación se utilizan los mismos procedimientos de derivación y composición que

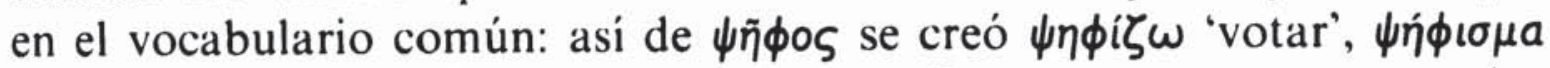
'voto, decreto'. Por último, en una tercera fase encontramos que neologismos jurídicos adquieren un uso común: así el verbo $\psi \eta \phi i \zeta \omega$ evolucionó del sentido técnico de 'votar' al valor material de 'contar', ávтrypaфń de 'escrito de defensa' (utilizado para rechazar la acusación del demandante) pasó a significar 'réplica', 'acción de transcribir' un escrito.

\section{Redacción de los articulos}

Para la distribución de un artículo hemos tenido en cuenta todos los datos posibles, tanto gramaticales como institucionales o legales, que ofrecía cada término. $\mathrm{Y}$ dos son las situaciones con que nos hemos encontrado:

1) Cuando una palabra tiene una sola acepción han primado, fundamentalmente, los valores sintácticos, tratando de reflejar todas las 
construcciones que aparecen en los textos. Para la distribución nos hemos servido de los números árabes (que separamos por punto y aparte) $\mathrm{y}$ de las letras minúsculas (que separamos por punto y coma). El número 1 lo reservamos para la definición del término, cuando se registra,

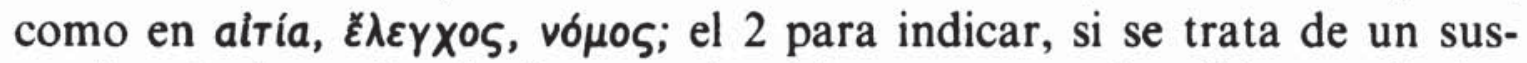
tantivo, la dependencia de un verbo (a), otro sustantivo (b), un adjetivo (c) o una preposición (d), o para indicar el uso absoluto, si se trata de un verbo (aquí incluimos las construcciones de participio sustantivado, a no ser que su extensión requiera uno propio. Si el empleo absoluto es resultado de la elisión de algún miembro, lo situamos en el apartado correspondiente, precedido de $s c$.); el número 3 o el que corresponda en cada caso se destina a los empleos del término en unión u oposición a otros términos jurídicos; el uso con genitivo ocupa siempre un número, en el caso de un sustantivo o adjetivo, clasificándose, si es posible, sus valores; si es un verbo, cada complemento ocupará un número; las oraciones completivas de infinitivo, $\delta \tau \iota, \dot{\omega} \varsigma, \varepsilon l$ e interrogativa indirecta, por su importancia en la gradación sintáctica requieren siempre un número independiente. Veamos algunos ejemplos:

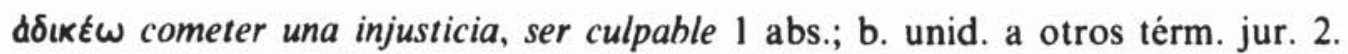
part. sust. 3. c. preps. 4. c. ac. int. 5. c. ac. de pers. 6. c. ambos.

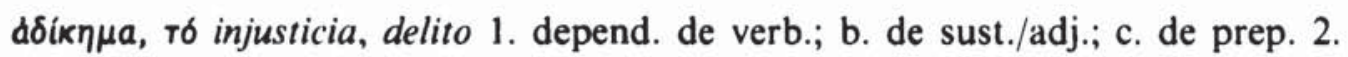
c. adj. 3. c. part. 4. unid. a otros térm. jur. 5. c. gen. subj.; b. c. gen. obj. 6.

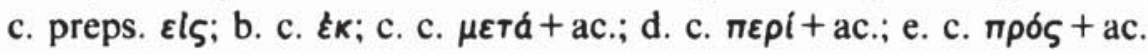

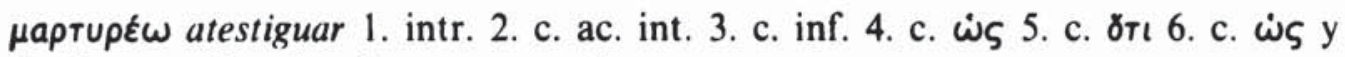
ठтı 7. c. or. de relat. 8. c. part. pred.

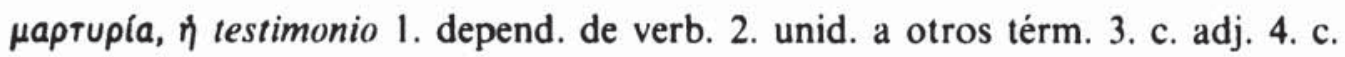
gen. de pers. 5. c. preps. 6. c. ac. int. 7. c. $\dot{\omega} \varsigma$ 8. c. ठัт 9. c. $\dot{\omega} \varsigma$ у $\delta_{T \iota} 10$. c. $\varepsilon l$ 11. c. Tís.

Al hacer estas divisiones no nos guía un criterio puramente formalista, sino la intención de resaltar, a través de ellas, los valores estilísticos de la lengua demosténica. Con los datos, que se repiten a lo largo de tantos términos, se podrían confeccionar trabajos reveladores en este sentido, como hace B. Gaya Nuño con las oraciones de infinitivo en su obra Sobre un giro de la lengua de Demóstenes (C.S.I.C., Madrid 1959),

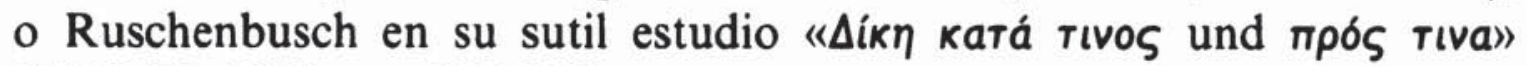
(ZRG 86, 1969, pp. 386-396).

En otras ocasiones, la numeración responde a las necesidades pro-

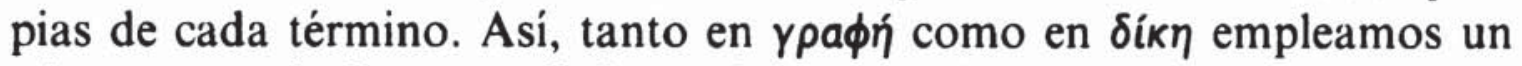
número para indicar sus distintos tipos (12 y 23 respectivamente). Igualmente, en $\delta i ́ k \eta$ registramos un número para su uso adverbial, obtenien- 
do 47 construcciones en su acepción de 'juicio' y 18 en la de 'castigo'.

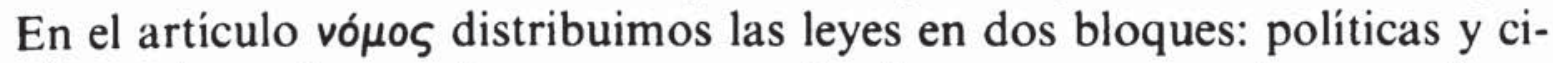
viles, asignando a cada una o grupo de ellas que versen sobre un mismo tema un número, con lo que sumamos 26 leyes políticas y 37 civiles.

En fin, la numeración, independientemente de su estructura sintáctica, puede matizar en cierto sentido la acción verbal o nominal, añadiendo información con base en el sujeto o en el objeto. Así lo vemos en:

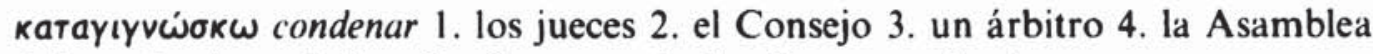
5. los miembros de la tribu 6. un particular 7. uno, a si mismo.

$\psi \eta \phi i \zeta o \mu a i$ votar 1. la Asamblea 2. los jueces 3. los demotas 4. los frateres 5. el Consejo.

$\delta \tilde{n} \mu \mathrm{S}_{\mathrm{S}}$ pueblo, Asamblea del pueblo 1. funciones juridicas 2. funciones legislativas.

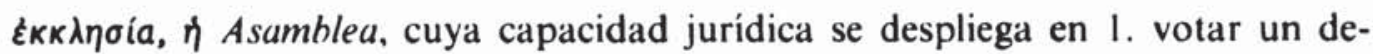
creto previo 2 . ratificar leyes 3 . conceder ciudadanía 4 . acusar a militares 5 .

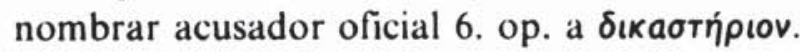

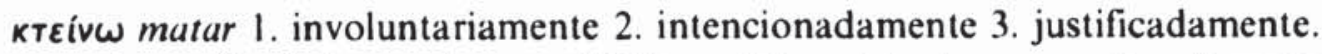
$T(\mu \omega$ pía castigo 1. impuesto por el tribunal 2. registrado en una ley. 3 propuesto en un decreto.

прокаһє́ода dirigir un requerimiento al oponente para solucionar algún conflicto 1. en gener. 2. para obtener un testimonio 3. para someter a tortura a un esclavo 4. para prestar juramento 5. para abrir un testamento 6. para abrir un contrato 7. para trasladar un problema a una magistratura extranjera 8 . para transmitir una trierarquia 9. para recurrir a un arbitraje 10. para cobrar un

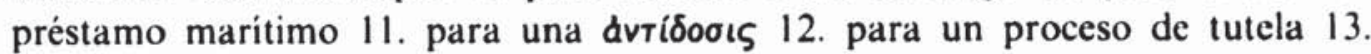
para demorar una votación de demotas 14. para un pacto de retroventa o derecho de redención 15. para un proceso por falso testimonio.

A veces definimos el término a lo largo de la distribución, como en:

ouvoıḱ́ $\omega$ convivir, estar casado con 1. def. 2. suj. la mujer 3. suj. el hombre 4 .

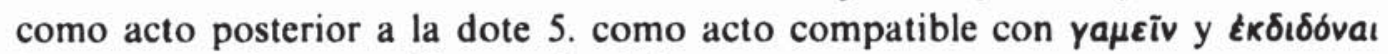

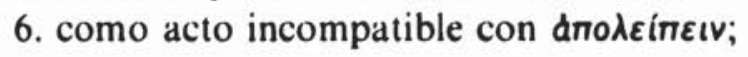

o en:

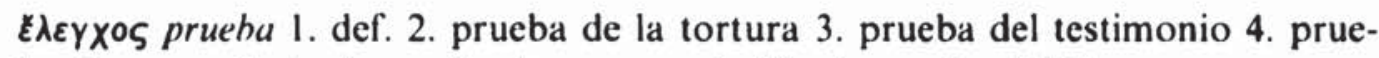
ba de un contrato 5. prueba de argumentación 6. prueba del tiempo.

En ocasiones, por último, incluimos gráficos, con la representación

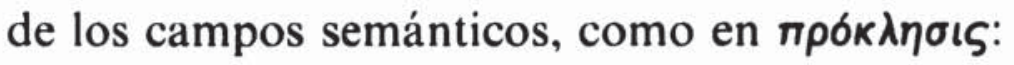




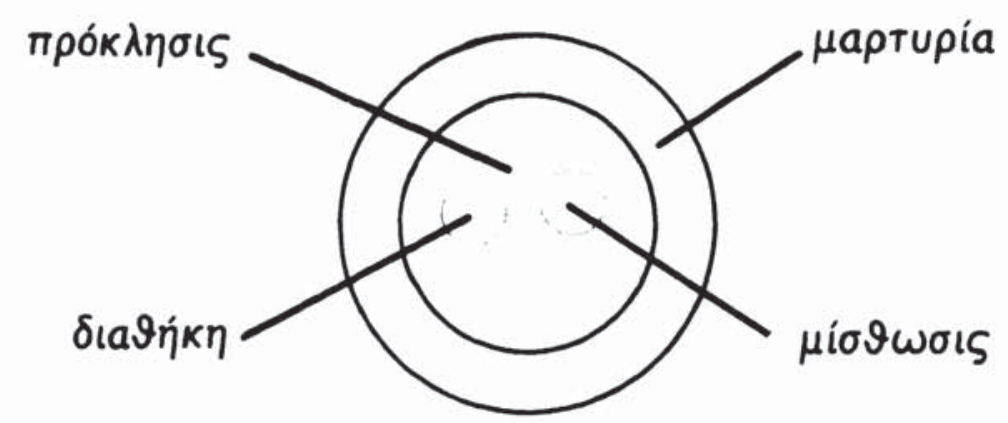

2) Cuando una palabra posee dos o más acepciones, utilizamos los números romanos para su distribución, reservando los números árabes para las mismas funciones que desempeñaban en el apartado anterior. Con número romano marcamos también la diferencia entre voz activa y voz media:

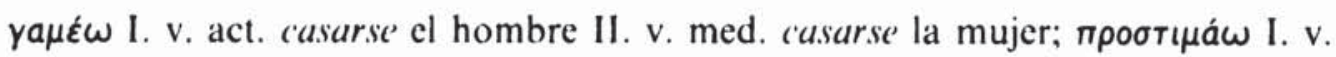
act. imponer pena suplementaria, el juez. II. v. med. proponer pena suplementaria, el demandante; ùnoтi $\eta \eta \mu$ I. v. act. dar en garantía II. v. med. recihir en garantia.

Cotejando nuestro Léxico con los grandes diccionarios generales, como el Liddell-Scott-Jones o el Diccionario Griego-Español (en sus dos

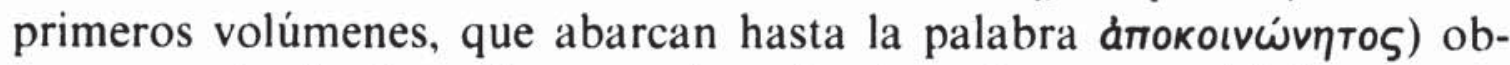
servamos dos hechos: 1) en muchos términos de nuestro Léxico encontramos acepciones que no se recogen en éstos, aunque sí se señale su valor jurídico; 2) de otros, su única acepción, naturalmente jurídica, no es advertida.

Del primer caso veamos algunos ejemplos:

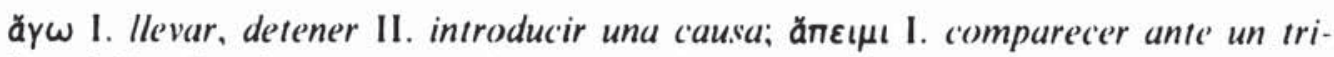

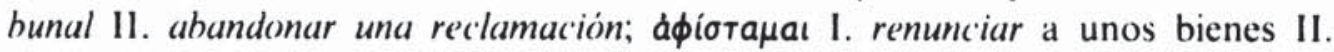
emanciparse un esclavo III. ser expulsado de un arrendamiento; $\varepsilon \pi \xi \xi \varepsilon \iota \mu$ у $\varepsilon \pi \varepsilon \xi$ -

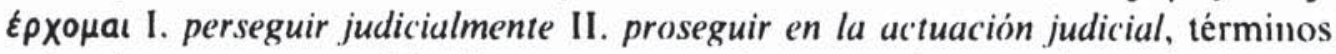
todos éstos cuya acepción II no aparece en los manuales anteriores; de $\varepsilon \lambda \hat{\varepsilon} \gamma \times \omega$ falta la primera: I. aportar una prueha II. poner a prueba. En otros ejemplos se confunden los dos valores: anodoyia I. defensa, medio de defensa II. discurso de

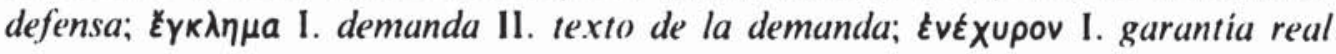
II. la prenda, propiamente dicha; $\psi \eta \tilde{\phi} \circ \varsigma$ I. guijarro para votar, de ahi, voto II. acto de votar, votación.

El artículo ßouגí en L.S.J. o en el Bailly alude sólo al 'Consejo de los Quinientos', sin apenas referencia al del Areópago; nosotros lo distribuimos así:

1. Consejo de los Quinientos 1. enclave geográfico 2. con competencias menores que un tribunal 3 . encargado de juzgar a los que retienen fondos públicos

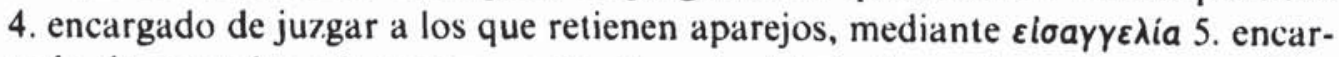
gado de proceder a la tortura; y II. Consejo del Areópago 1. enclave geográfico 2. competente en homicidios y heridas 3. competente en asuntos de Estado. 
Para terminar de cotejar, transcribiremos la cita literal de un térmi-

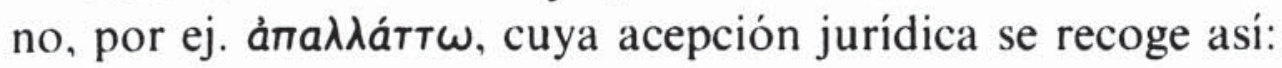
L.S.J.
I. act. set free, deliver from a thing... 4. in Law, give a release, dis- charge. II Pass. and Med., to he free or released from a thing.
D.G.E. I. gener. en v. med. -pas., c. gen. de abstr., retirarse, renunciar d) esp. jur. retirarse, renunciar a las acusaciones.
L.J.D. I. liherarse de un prestamista II. descargar de una obligación (unid.

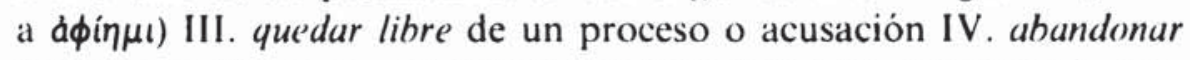 un proceso V. ser manumitido un esclavo (pas.) VI. separarse la mu- jer.

Del segundo caso, términos jurídicos cuya acepción jurídica no es advertida, destaquemos algunos ejemplos: el part. sust. $\delta$ ßouגó $\mu \varepsilon v o s$, "el que lo desee', hace referencia a toda persona capacitada para presentar una acusación pública; incluimos yóvara 'rodillas', por ser utilizado el

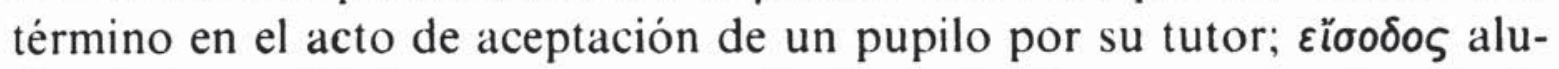
de a la 'entrada' de un proceso en el tribunal y है $\rho x o \mu a \iota$ se emplea en algunas ocasiones con el valor de 'recurrir a juicio'; $\delta \rho a \omega$ adquiere el sen-

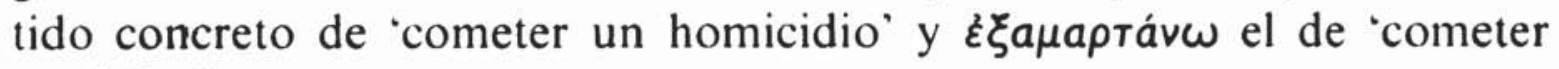
un delito'.

En cuanto a los signos utilizados, además de los números y letras, encontramos los siguientes: los paréntesis, que pueden tener una doble finalidad, o desarrollar el contenido de un contexto o, puestos en una cita, indicar que ésta ha aparecido o aparecerá en otro apartado (una cita puede repetirse tantas veces como estructuras sintácticas o semánticas presente); los corchetes, situados al final del artículo, añaden infor-

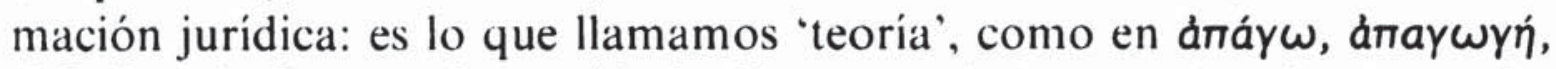

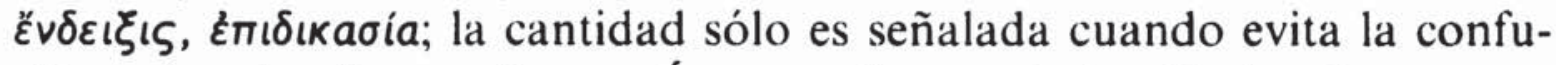

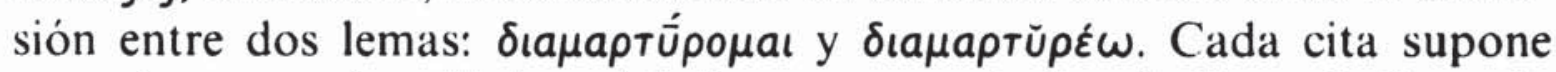
tres números: a los clásicos del discurso y capítulo añadimos la línea de este último, con el fin de facilitar su localización; el adverbio bis tras una cita indicada que el término aparece dos veces; si la cita carece del tercer número, el término en cuestión no es recogido, pero sí su concepto; cuando la cita procede de una ley u otro documento insertado en los discursos, la precedemos de doc. Las Cartas se recogen con número romano, los Proemios con la letra p. y los discursos se numeran según la edición de H. Butcher-W. Rennie, Orationes, 4 vols., Oxford 1903-1931 (no reproducimos los corchetes cuando los hay, aceptando por tanto las conjeturas del editor; en cambio, en algunos pasajes, concretamente en siete, hemos adoptado lecturas propias).

\section{Antonio Ramón Navarrete Orcera}

